\title{
Sobre gestión de medios: elogio a la trascendencia
}

Manuel Goyanes ${ }^{1}$

Recibido: 2015-11-05

Enviado a pares: 2015-11-11
Aprobado por pares: 2015-12-17

Aceptado: 2016-01-13

DOI: 10.5294/pacla.2016.19.3.8

Para citar este artículo / to reference this article / para citar este artigo Goyanes, M. (2016). Sobre gestión de medios: elogio a la trascendencia. Palabra Clave 19(3), 868-892. DOI: 10.5294/pacla.2016.19.3.8

Al tomar por objeto un mundo social en el que uno se halla comprendido, se obliga a tropezar, bajo una forma que podría llamarse dramatizada, con una cierta cantidad de problemas epistemológicos fundamentales, ligados todos ellos a la cuestión de la diferencia entre el conocimiento práctico y el conocimiento erudito, y especialmente a la dificultad particular de la ruptura con la experiencia nativa, originaria, y de la restitución del conocimiento obtenido al precio de dicha ruptura. Uno sabe qué obstáculo representa para el conocimiento científico tanto el exceso de proximidad como el exceso de distancia, y la dificultad de instaurar esa relación de proximidad rota y restaurada que, al precio de un largo trabajo sobre el objeto pero también sobre el sujeto de la investigación, permite integrar todo aquello que no se puede saber a menos que uno lo sea y todo aquello que no se puede o no se quiere saber porque uno lo es.

Pierre Bourdieu

\section{Resumen}

El presente artículo problematiza la estructura del relativamente reciente subcampo académico científicamente denominado gestión de medios (media management). Concretamente, se articula en torno a la reflexión crítica sobre lo que conceptualizo como CADI, es decir, cuatro dimensiones es-

$1 \quad$ Universidad Carlos III de Madrid, España.mgoyanes@hum.uc3m.es 
tructurales que modulan el "espíritu" de su forma: 1) campo, 2) autonomía, 3) dominación técnico-práctica y 4) identidad. El CADI, que usualmente es retratado como modelo de la buena práctica investigadora, se configura en este artículo como un estado de confort académico, en el que una serie de disposiciones, prácticas y habitus socializados por la comunidad de gestión de medios podrían obstaculizar y oprimir el progreso académico hacia otras alternativas intelectuales y modus de descubrimiento y verificación científica. Por ello, propongo el concepto de 'ruptura trascendente' como estrategia de emancipación individual y disciplinaria del régimen ideológico estructural del subcampo a través de tres versiones: ruptura teórica, ruptura funcional y ruptura epistemológica.

\section{Palabras clave}

Ciencias de la comunicación; gestión de medios; media management; trascendencia; dominación (Fuente: Tesauro de la Unesco). 


\section{On Media Management: In Praise of Transcendence}

\section{Abstract}

This article questions the structure of the relatively recent academic subfield known scientifically as media management. In doing so, it takes a critical look at what the authors conceptualize as CADI (Spanish acronym), which stands for four structural dimensions that modulate the "spirit" of its form: 1) field, 2) autonomy, 3) technical and practical domination and 4) identity. CADI, which usually is portrayed as a model of good research practice, is described in this article as a state of academic comfort in which a series of rules, practices and habitus socialized by the media management community might actually obstruct and repress academic progress toward other intellectual alternatives and modes of discovery and scientific verification. For that reason, the author proposes the concept of "transcendent rupture" as a strategy to achieve individual and disciplinary emancipation from the structural ideological regime of the subfield through three versions: theoretical rupture, functional rupture and epistemological rupture.

\section{Keywords}

Communication sciences; media management; transcendence; domination (Source: Unesco Thesaurus). 


\section{Sobre gestão de meios: elogio à transcendência}

\section{Resumo}

Este artigo problematiza a estrutura do relativamente recente subcampo acadêmico cientificamente denominado gestão de meios (media management). Em especial, articula-se em torno da reflexão crítica sobre o que se conceitua como CADI, isto é, quatro dimensões estruturais que moldam o "espírito" de sua forma: 1) campo, 2) autonomia, 3) dominação técnicoprática e 4) identidade. O CADI, que usualmente é retratado como modelo de boa prática investigadora, configura-se neste artigo como um estado de conforto acadêmico, no qual uma série de disposições, práticas e hábitos socializados pela comunidade de gestão de meios poderiam impedir e oprimir o progresso acadêmico a outras alternativas intelectuais e modus de descobrimento e verificação científica. Por isso, propõe-se o conceito de "ruptura transcendente" como estratégia de emancipação individual e disciplinar do regime ideológico estrutural do subcampo por meio de três versões: ruptura teórica, ruptura funcional e ruptura epistemológica.

\section{Palavras-chave}

Ciências da comunicação; gestão de meios; media management; transcendência; dominação (Fonte: Tesauro da Unesco). 


\section{Introducción}

En términos generales, una de las principales finalidades de todo campo es el de lograr su madurez intelectual a través del desarrollo de cierta autonomía frente a otros campos. Esta genuina teleología de los campos entraña, sin embargo, una doble complejidad: por un lado, mantener la "esencia" y el "espíritu" original de su creación como campo original, y por otro, enriquecerse (o empobrecerse) de las influencias externas procedentes de otros campos más próximos o más lejanos (lo que en la jerga académica moderna se configura como cross-fertilization). La lucha por la dominación del campo y la jerarquización de sus agentes ha sido una constante en las ciencias sociales, intensificada durante las últimas tres décadas como resultado de la creciente especialización en subcampos.

Es este el caso particular de la gestión de los medios o media management, subcampo a caballo entre la gestión pura y los media and communication studies. Subcampo híbrido que, sin embargo, ha logrado constituirse con cierta autonomía a través del desarrollo de espacios, instituciones e identidad soberana relativamente respetada. La especialización y la socialización de unos esquemas disciplinarios comunitarios han logrado, de este modo, el desarrollo y la aceleración de unos conocimientos más profundos y contrastados sobre ciertos aspectos que ni la gestión pura ni los media and communication studies habrían alcanzado por sí solos. Asimismo, la pertenencia colectiva al subcampo específico ha reportado pingües beneficios a sus integrantes: por un lado, ha creado una escuela intelectual y una identidad investigadora concreta, y por otro, quizá lo más importante, ha incrementado la productividad de su tribu (en particular respecto a publicaciones en revistas científicas). Sin embargo, uno se puede preguntar hasta qué punto estos esquemas socializados a lo largo de los años en nuestro subcampo de especialización ya maduro realmente conducen a unos resultados académicos noveles, imaginativos e influyentes.

A pesar de que cierto grado pertenencia a un campo o un subcampo es siempre necesario así como positivo, la asimilación de ciertos esquemas, jerarquías y estructuras tradicionales (en general de dominación) conlleva ciertos peligros que, como espacio científico, debemos resaltar, denunciar y 
superar. Por ejemplo, la confusión entre campo y objeto, la inclinación hacia determinadas epistemologías y teorías funcionalistas, la preponderancia técnica e instrumental frente al desarrollo teórico, así como la influencia de lo que denomino contribución no sancionada, son algunos de los esquemas y peligros disciplinarios que hemos ido adquiriendo a lo largo del proceso de maduración del subcampo. Concretamente, estos esquemas socializados a lo largo del proceso de cultivo de la gestión de los medios han supuesto el advenimiento actual de un estado académico de confort que, aparentemente, se configura como un juego ganar-ganar: el subcampo avanza mientras sus integrantes son científicamente productivos.

En el presente artículo, exploro los posibles problemas del estancamiento en la zona de confort de la gestión de los medios mediante la descripción crítica de cuatro dimensiones estructurales (campo, autonomía, dominación e identidad [CADI]), que la configuran como subcampo de especialización. Concretamente, discuto los esquemas académicos que durante su etapa de madurez disciplinaria han sido ampliamente socializados por la mayoría de sus integrantes como agentes heterónomos. El aprendizaje de las "normas" en cada una de las dimensiones estructurales configura un espacio académico cómodo, acotado y seguro en el que la productividad y la visibilidad de sus integrantes podría superponerse frente a la proposición de aproximaciones más innovadoras, desafiantes o radicales.

El objetivo del presente artículo es el de animar, por ello, una conciencia crítica partiendo de un profundo análisis de los esquemas mentales y académicos de las cuatro dimensiones estructurales que configuran nuestra área. Normas dadas por hechas, racionales, naturales y aparentemente sustantivas para el progreso como espacio de especialización que, sin embargo, articulan y reflejan esquemas dominantes acerca del modelo de descubrimiento y verificación científico, que pueden ser peligrosos para el desarrollo de conocimiento, así como elemento de inhibición de una investigación más imaginativa e interesante. Por ello, propongo la ruptura trascendente como estrategia alternativa a los actuales esquemas desarrollados a través de las cuatro dimensiones estructurales. La ruptura trascendente anima y propone a los investigadores la modificación del objeto, la 
experimentación con otros campos teóricos alejados del área de especialización, así como la consideración de epistemologías procedentes de otros terrenos intelectuales.

La línea argumental de mi análisis se establece, a grandes rasgos, con las actuales reflexiones que cuestionan, problematizan y discuten la importancia de la gestión de los medios como subcampo de especialización. En este sentido, diferentes autores ya se han preocupado y pronunciado sobre los problemas y las dificultades del progreso académico de la gestión de los medios como subcampo relativamente autónomo (Campos-Freire, 2010; Küng, 2007; Goyanes, 2013; Artero, 2015). Mi enfoque no es primeramente ese, si no el análisis estructural de la gestión de los medios como subcampo a través del estudio de los esquemas socializados que gobiernan y condicionan la trayectoria investigadora de los integrantes mediante el estudio de su campo, autonomía, dominación e identidad. Para ello, utilizo la metáfora del "estado de confort académico" como elemento aglutinador que confiere la gestión de los medios y a sus integrantes una total, aunque cuestionable, comodidad y seguridad académica, institucional e intelectual.

El artículo se articula del siguiente modo. Inicialmente, describo las dimensiones estructurales que conforman la gestión de los medios como subcampo de especialización y que la dotan, a posteriori, de su controvertido estado de confort académico actual. En este punto, explico su configuración, así como las interrelaciones existentes entre cada una de las dimensiones. Asimismo, discuto las principales ventajas de la pertenencia al subcampo, así como, fundamentalmente, las principales desventajas a la hora del planteamiento de una investigación alternativa. A continuación, propongo la ruptura trascendente como estrategia de emancipación de los esquemas paradigmáticos y, finalmente, analizo brevemente la integración de la ruptura trascendente dentro de los estudios generales sobre gestión de medios.

\section{La zona de confort académico de la gestión de los medios}

De modo muy similar a otros espacios académicos, la gestión de los medios como subcampo se ha ido especializando a través de la focalización 
en determinadas corrientes teóricas, aproximaciones metodológicas y esquemas de cientificidad. Durante su evolución histórica ha ido adquiriendo una visión particular, así como ha socializado a sus integrantes con unas formas académicas representativas de su "escuela". La investigación sobre organización de la ciencia ha descrito estas visiones o esquemas estructurales de un campo a través de diferentes conceptualizaciones: disciplinas, tribus, comunidades epistemológicas, etc. Particularmente, acudiendo a la terminología de Kuhn (1970), el concepto de 'paradigma' ha sido ampliamente el término aglutinador de todas ellas, designando los compromisos y las prácticas compartidas por una comunidad de científicos. Por un lado, los teóricos, ontológicos y credenciales, y por otro, aquellos compromisos que hacen referencia a la aplicación de la teoría y a los modelos de soluciones de problemas.

El presente artículo no se interesa por el concepto de 'paradigma', stricto senso, sino más bien por las más estrechas estructuras que configuran un subcampo (en este caso la gestión de los medios) como un espacio académicamente maduro y con instituciones soberanas. Esta estructura, en nuestro caso, se modela a través de cuatro dimensiones que, de modo general, ofrecen a la gestión de los medios su situación disciplinaria de madurez y, a posteriori, su problemático estado de confort académico: campo, autonomía, dominación e identidad. Lo que discuto es el aparente progreso de nuestra escuela académica a través de la problematización y crítica de los supuestos esquemas racionales desarrollados.

Deliberadamente, utilizo la conceptualización de "estado de confort académico" como metáfora, con el objetivo de evitar una precisa definición o estricta descripción empírica (Brown, 1976; Morgan, 1980). Mi objetivo, por tanto, no es el de contribuir al progreso de nuestra área a través de una profunda idealización de mi investigación. Por el contrario, lo que trato es de desafiar las ideas dominantes que configuran estructuralmente la gestión de los medios y proponer una estrategia que busca la adquisición de mayor trascendencia académica como intelectual. Concretamente, utilizo la metáfora del estado de confort académico con el objetivo de describir cuatro dimensiones estructurales interrelacionadas que conforman la gestión de los medios: 
- Campo: la gestión de los medios como subcampo a caballo entre el campo de la gestión pura y el campo de los media and communication studies.

- Autonomía: la gestión de los medios como subcampo relativamente emancipado de sus campos originales.

- Dominación: la gestión de los medios como subcampo dominado por un modelo técnico-práctico de producción científica.

- Identidad: la gestión de medios como escuela o tradición disciplinaria específica de sus integrantes.

La metáfora del estado de confort implica, por tanto, un fuerte y estricto compromiso con un determinado esquema de cientificidad, una lógica práctica de investigación científica y hábitos para el desarrollo competente e identitario. Este reconocimeinto colectivo de la hoja de ruta científica ofrece los recursos funcionales elementales que permiten a los miembros de la tribu incrementar exponencialmente su producción científica y su carrera investigadora.

La zona de confort se trata, originalmente, de un concepto procedente de la psicología emocional, que designa ese espacio, no necesariamente físico, en el cual una persona se siente en especial cómoda, segura y consciente de los límites que ella misma se impone. Se trata de un conjunto de prácticas, estilos, tácticas y tradiciones que se aplican habitualmente y que configuran un espacio de control individual. Sin embargo, esta situación aparentemente beneficiosa tiende a interponer barrearas contra alternativas transgresoras o radicales que desestabilicen su estado de confort actual, aunque se traten de medidas orientadas al desarrollo, en el caso de nuestro análisis, orientadas al desarrollo de nuestro subcampo. A lo largo de los siguientes apartados, analizaré las cuatro dimensiones estructurales que conforman la gestión de los medios y sus interrelaciones, así como los principales esquemas (teóricos, metodológicos, identitarios, en definitiva, académicos) que las configuran y que sitúan a la disciplina en un problemático estado de confort. 


\section{Campo}

La primera dimensión estructural que configura la gestión de los medios es la articulación de su campo. El campo hace referencia a un espacio de acción social y de influencia en el que confluyen diferentes relaciones sociales determinadas y que se configuran con relativa autonomía frente a otros campos o la sociedad en su conjunto (Bourdieu, 1988). Del mismo modo, el campo se articula a través de diferentes agentes jerárquicos que luchan sostenidamente para ocupar las posiciones dominantes. En la gestión de los medios, se configura como un subcampo híbrido a caballo entre el campo de la gestión pura y el campo de los communication and media studies. Cada uno de estos campos ofrece, por tanto, unos esquemas y hábitos que configuran la autonomía e identidad de la gestión de los medios, así como unos agentes (teóricos, epistemológicos o metodológicos) determinados que luchan por el dominio del subcampo.

A la hora de analizar la gestión de los medios, es preciso, por tanto, la articulación teórica de los dos campos de los que recibe sus esquemas y prácticas académicas y que configuran su forma como disciplina científica. Por un lado, el campo de la gestión pura, y por otro el campo de los communication and media studies. Finalmente, la hibridación que configura el subcampo de la gestión de los medios:

- Gestión pura: campo conformado por diferentes subcampos que analizan las organizaciones empresariales, con el objetivo de identificar las estructuras y prácticas que maximicen su capacidad para sobrevivir y prosperar bajo ciertas condiciones de mercado.

- Communication and media studies: campo conformado por diferentes subcampos que analizan los fenómenos sociales relacionados con la comunicación (no necesariamente humana), así como los media como territorio de observación privilegiado para el estudio de la sociedad y sus mutaciones.

- Gestión de medios: subcampo híbrido conformado por diferentes subcampos del campo de la communication and media studies, así como diferentes subcampos del campo de la gestión pura, que tiene como objeto el análisis de los media en cuanto a organización, 
privilegiando a unos agentes dominadores de herencia técnicopráctica a través de epistemologías positivistas heredadas del campo de la gestión pura.

El elemento fundamental a la hora del establecimiento de la gestión de los medios como subcampo es la distinción entre campo/objeto y el privilegio del objeto como configuración del campo. La gestión de los medios tiene como objeto los media, pero tan solo a los media en cuanto organización (es decir, articulados ciegamente a través de tres de sus formas fundamentales de organización: prensa, radio, televisión y, quizá, el cine y los nuevos medios), pero no como (principalmente) espacios o territorios de análisis de las relaciones humanas "en" o "a partir de" los media o como agentes de estudio de la sociedad y sus posibles mutaciones. Por tanto, la configuración del propio objeto en cuanto a únicamente organización como elemento de análisis del subcampo implica la importación de los agentes dominadores que configuran al campo matriz, es decir, de la gestión pura, y el empobrecimiento de la tradición heredada del campo de los communication and media studies como campo de análisis de los media en cuanto espacio de análisis social. Por tanto, la dominación técnico-práctica a través de una visión funcional del subcampo prevalece frente al análisis más teórico o sociológico de los media.

La dominación de la organización en cuanto objeto en los estudios sobre gestión de medios implica, por tanto, una configuración teórica que privilegia una aproximación del campo de la gestión pura y sus epistemologías dominadoras, lo cual no supone, sin embargo, la no inclusión de perspectivas teóricas multidisciplinarias procedentes de otros campos, como la sociología, psicología o economía u otras aproximaciones al objeto procedentes de otros subcampos, por ejemplo los estudios en periodismo. Por el contrario, la inclusión de estas perspectivas teóricas multidisciplinarias, así como la asimilación de diferentes aproximaciones al objeto, se produce temporalmente, pero de tal forma que su absorción tiende a reproducir los esquemas dominadores de la gestión pura.

Es en este confluir teórico y de aproximaciones al objeto donde el subcampo se divide en compartimentos intelectuales estanco, que buro- 
cratizan el trabajo investigador especializado en gestión de medios, donde medios significa organización y organización campo de gestión pura. El investigador se configura como un experto en temas hiperespecializados en el que se siente cómodo y seguro y donde la división del trabajo adquiere una significación identitaria y colectiva y que impide los movimientos radicales interdisciplinarios. Surge, de este modo, el investigador-especialista acomodado y seguro de sus limitaciones, encargado de una especificad concreta del trabajo investigador dentro del subcampo: investigador-teórico, investigador-estadístico, investigador-metodológico, en gestión de medios. El subcampo adquiere una identidad soberana y una relativa autonomía (aunque heredada) frente a los campos matriciales, de tal modo que los esquemas académicos socializados permiten el aparente progreso del subcampo y un estado de confort tanto de sus integrantes, ya inmersos dentro de una red superior de relaciones dentro y fuera del subcampo, como de la propia gestión de medios. Ello impide, paralelamente, el cuestionamiento de sus resultados y un tipo de investigación alternativa.

\section{Autonomía}

Como todo campo (o subcampo), la gestión de los medios busca su propia autonomía, es decir, la articulación de sus propias leyes y la búsqueda de una posición dentro del orden científico social. Su configuración como subcampo híbrido le proporciona, sin embargo, una doble condición: la lucha por mantener la autonomía como subcampo soberano, y las numerosas influencias externas de los dos campos originarios (fundamentalmente) de los que hereda su estructura como subcampo: la gestión pura y los communication and media studies. Esta doble condición de la gestión de los medios implica, por tanto, la configuración de un espacio social estructurado, un campo de fuerzas fijas de dominantes y dominados, relaciones de desigualdad que intentan transformar o conservar el subcampo y, consecuentemente, la aplicación de estrategias en su beneficio.

La relativa autonomía de la gestión de los medios como subcampo se articula a través de la hibridación de los dos campos de los que originalmente procede. La hibridación de la gestión de los medios resulta en aspectos positivos y negativos para su progreso disciplinario: por un lado, implica la asimilación de diferentes aproximaciones (teóricas, metodológicas y 
epistemológicas fundamentalmente) heredadas de los dos campos originales (u otros externos) que lo enriquece (o empobrece) como subcampo; y por otro, la contribución no sancionada de integrantes de los campos o subcampos originales (u otros) en el subcampo de la gestión de los medios. Esta configuración que, a priori, parece muy abstracta, se puede explicar más sencillamente.

La gestión de los medios se configura como un subcampo a caballo entre el campo de la gestión pura (y todos sus subcampos) y los communication and media studies ( $\mathrm{y}$ todos sus subcampos). En cada uno de los subcampos que componen la gestión pura y los communication and media studies, hay dominadores y dominados según los valores internos del propio campo. De este modo, un investigador del campo de la gestión pura será un "buen investigador" en cuanto es sancionado como tal por los buenos investigadores de su propio campo. Esta sanción se configura necesariamente de forma circular. Sin embargo, la heteronomía empieza cuando una contribución de un aparente especializado en gestión pura contribuye en apariencia de modo trascendental a la gestión de los medios, cuando nunca ha sido sancionado ni reconocido dentro de su propio campo como buen investigador.

La autoridad que proviene de la especialización ha desembocado en una contribución que, en múltiples ocasiones, no ha sido sancionada por el campo original. Esta es la ambivalencia de la especialización en gestión de medios: la adquisición de notables descubrimientos basados en un análisis minucioso de una pequeña porción de realidad y la aparente contribución de resultados basados en la especificidad que el campo original da por hecho, pero que el campo al que se anexiona desconoce por completo. Se podría concluir, por tanto, que en la gestión de los medios confluyen una amalgama de "sabios ignorantes"; es decir, hombres de ciencia conocedores de su porciúncula de universo, pero que ignoran cuanto no entra dentro de la especialidad (Ortega y Gasset, 1929).

La relativa autonomía de la gestión de los medios como subcampo proporciona a sus integrantes un fuerte poder de identificación, fundamentalmente a través de la configuración de diferentes redes de interacción 
académica y la socialización de sus esquemas y hábitos desarrollados durante años. Esta autonomía, que se fundamenta asimismo a través de la hibridación de los esquemas de los dos campos donde tiene origen, sitúa el subcampo en su madurez intelectual, que implica la consecución de unos espacios soberanos. En ellos sus integrantes despliegan sus esquemas tradicionalmente socializados, se sienten cómodos y no se cuestionan la trascendencia de sus resultados para el campo social en su conjunto.

\section{Identidad}

La pertenencia al subcampo de la gestión de los medios proporciona a sus integrantes unas categorías que ayudan a organizar la realidad investigadora. Como categorías entiendo las estructuras invisibles que organizan lo percibido y determinan lo que se ve y lo que no se ve; es decir, los lentes desarrollados que se articulan a través de nuestra educación, experiencia científica y disposición intelectual (Bourdieu, 1996). Estas categorías perceptivas focalizan al investigador en el subcampo, reducen el caos inherente a todo saber social y dota a sus integrantes de cierta identidad escolástica.

La identidad proporciona al subcampo y a sus integrantes un espacio de integración individual o colectivo, colabora a la autonomía disciplinaria, así como desarrolla una fuerte autodefinición integral del subcampo como de los propios integrantes (yo pertenezco a la red de gestión de medios y trabajo sobre estrategias multiplataforma en la televisión). El elemento de pertenencia al subcampo crea, por tanto, patrones o modelos de identidad a través del desarrollo de diferentes perfiles de investigador a modo de marca personal y el desarrollo de clústeres de interacción individual o colectiva (congresos, conferencias o workshops sobre diferentes sub-temáticas dentro del subcampo), donde los integrantes se reafirman como partes integrantes del subcampo que, asimismo, reafirman el carácter autónomo y soberano de la identidad del subcampo.

La identidad de la gestión de los medios se configura, por otra parte, a través de un fuerte contenido ideológico heredado de la gestión pura que impregna todas sus dimensiones estructurales. El concepto de ideología’ es un término ampliamente utilizado en ciencias sociales, la mayoría 
de las veces con una connotación peyorativa fruto de la tradición marxista. Mi visión de ideología retoma una aproximación sustancialmente crítica, fundamentada en la formación de una imagen positiva y atractiva que legitima ciertos intereses y un ordenamiento social específico (Alvesson, 1987). La identidad de la gestión de los medios hereda de la gestión pura sus agentes dominadores en cuanto trata la "organización" como objeto, asimilando una visión técnico-práctica de sus resultados y reproduciendo un mensaje esencialmente funcional, fundamentado en un modo particular de descubrimiento y producción científica basado en la técnica y la medición cuantitativa. Bajo esta ideología, prevalece lo instrumental o lo técnico frente a lo trascendente o teórico, efecto que conlleva unos juicios de valor (no referencia a valores) públicos (y políticos) acerca de la propia disciplina y que, desde un punto de vista científico, deberían estar totalmente neutralizados (Weber, 1946).

La adquisición y sanción externa de una identidad soberana confiere a los integrantes de la gestión de los medios un hábitat o espacio intelectual acotado donde los miembros se autoconfirman y se sienten confirmados, cómodos y "en casa". La identidad de la comunidad, así como sus competencias adquiridas, conducen a la reproducción de unos esquemas académicos enteramente socializados y repetidos, que conducen a un estado de madurez disciplinario y a la conservación de lo adquirido frente a las suspicacias de lo extraño (en general agentes procedentes de otros campos) que perturban su identidad como subcampo soberano y relativamente autónomo. El estado de confort se adueña del campo e impide una evolución alternativa.

\section{Dominación}

La gestión de los medios se configura como un subcampo, cuyo objeto son los media en cuanto organización. El privilegio del objeto como elemento configurador del campo implica la importación de las teorías y epistemologías del campo de la gestión pura para el análisis específico de los media. Por tanto, la gestión de los medios hereda de la gestión pura su visión orientada a objetivos basada en el análisis e identificación de las estructuras y prácticas que maximicen la capacidad de supervivencia de las organizaciones 
(los media) bajo unas determinadas condiciones de mercado. Esta particular configuración del subcampo reproduce los agentes de dominación de la gestión pura, basados en la orientación técnico-práctica con la cuantificación de variables y el empleo de modelos estandarizados de descubrimiento y verificación científica ampliamente socializados por sus integrantes.

La dominación técnico-práctica mediante modelos socializados sobre el proceso de descubrimiento y verificación científica implica el desarrollo de patrones estándar sobre el progreso del subcampo. La estandarización es un producto de una secuencia codificada e interrelacionada de prácticas formuladas normalizadas que implican investigaciones estándar, procesos editoriales estándar, revisiones estándar y, de modo general, esquemas mentales estándar, es decir, modos estandarizados de pensar sobre lo que constituye la gestión de los medios y sus formas (Alvesson y Gabriel, 2013; Goyanes, 2015). Este tipo de dominación privilegia la diseminación del conocimiento incremental mediante la utilización de la técnica (a través de una aproximación hipotético-deductiva ${ }^{2}$ y el reduccionismo que implica la diseminación del conocimiento en un formato de entre 6000 y 8000 palabras: el journal.

Esta particular visión dominante de lo que se considera "buena investigación" se articula a través de cuatro dimensiones que modelan este fenómeno sobre cómo, en efecto, la producción del saber y el descubrimiento científico debe ser diseñado y desarrollado dentro del subcampo: 1) hiperfocalización, 2) detección de una laguna, 3) racionalismo y reduccionismo estadístico e 4) ingeniería estilística y gimnástica retórica. A continuación, describiré brevemente cada una de ellas. ${ }^{3}$

Hiperfocalización. Los investigadores planifican, diseñan y ejecutan una fuerte inversión en un tema hiperespecializado y no suelen abandonarlo hasta su efectiva y completa disección analítica. Este procedimiento académico permite el estudio, el análisis y la contribución incremental del impacto de múltiples variables y constructos sobre el objeto de estudio, así

2 Aunque su estructura también está abierta a cualquier aproximación metodológica estandarizada.

3 Para más detalle de la estructura de la estandarización, consulte el artículo de Goyanes (2015). 
como el diseño acumulativo de modelos empíricos. Como resultado, las investigaciones tienden a atesorar un profundo carácter idiosincrático a través de la utilización de una jerga reservada y unos procedimientos estilísticos, metodológicos y contributivos tribales, en la que sus integrantes se autoconfirman y se sienten cómodos.

Detección de una laguna. La detección de una laguna para ser cubierta exige el profundo escrutinio de la literatura previa sobre el subcampo. Los investigadores leen, reproducen y asimilan asunciones e ideas dadas por hechas. El notable incremento de este tipo de investigación incremental (sobre relaciones entre variables o constructos específicos) ha provocado en el establishment académico una cada vez mayor dificultad para proponer una laguna que no haya sido previa e empíricamente analizada. En este caso, la construcción retórica y social a partir de un ordenado y desordenado de la literatura previa permite desarrollar suficientemente una laguna creíble para motivar otro estudio.

Racionalismo y reduccionismo estadístico. Uno de los elementos clave a la hora de justificar la correcta opción metodológica se basa en el detalle extremo del procedimiento investigador, a través de una definición cristalina de los objetivos del artículo y una operacionalización de las variables siguiendo estudios previamente aceptados y publicados. La contribución empírica se basa en testar positivamente las hipótesis planteadas a través de técnicas estadísticas hiperrefinadas basadas en procedimientos de procesamiento de datos estandarizados y ampliamente adquiridos por el establishment. Con el fin de desarrollar una "gestión de datos" y un análisis estrictamente riguroso, los investigadores crean una historia ficcionada de sus actividades de investigación embelleciéndolas y racionalizándolas para situarlas a la altura de los ideales, muy lejanas, en cambio, del desorden y caos que caracteriza la mayoría de investigaciones, buenas y malas.

Ingeniería estilística y gimnástica retórica. Los artículos académicos estandarizados siguen un patrón estilístico y estructural bien conocido. La fórmula industrial estandarizada se basa, en general, en los siguientes ingredientes: detección del problema, conocimiento de estudios previos, explica- 
ción de los objetivos y metodología y, finalmente, justificación del estudio. Este planteamiento se articula y estructura en torno a cuatro preguntas clave: 1) ¿cuál es el problema?, 2) ¿qué es lo que sabemos (hasta ahora)?, 3) ¿qué es lo que vas a hacer y con qué medios? y 4) ¿por qué es importante?

El dominio de la estandarización como esquema de desarrollo y producción académico del subcampo implica la adquisición de un modelo que permite el progreso. A través de la estandarización tanto la gestión de los medios como sus integrantes adquieren unas fórmulas aceptadas por otros campos que permiten la autoafirmación como subcampo soberano y la identificación de sus integrantes con unas prácticas y esquemas de desarrollo intelectual ampliamente socializados por la academia en su conjunto. En consecuencia, el dominio de la visión técnico-práctica a través de la estandarización desencadena un estado de confort, donde el subcampo y sus integrantes se sienten cómodos y con control sobre el futuro productivo de ellos mismos, así como del subcampo. Sin embargo, en contrapartida, las alternativas (en general epistemológicas) no estandarizadas sufren para incorporarse al subcampo.

\section{Ruptura trascendente}

Estas son las cuatro dimensiones que estructuran la gestión de los medios y que la configuran como un subcampo maduro con unas prácticas y hábitos socializados, donde sus integrantes y la propia disciplina se siente cómoda y que, a posteriori, la sitúan en un estado problemático de confort académico que impide el propio cuestionamiento de la trascendencia de sus resultados en un sentido más amplio. Mi propuesta, orientada a la trascendencia, cuestiona los supuestos sobre los que se estructura el subcampo y plantea su ruptura a través de tres estrategias basadas globalmente en la modificación del objeto, la experimentación con otros campos teóricos, así como la consideración de epistemologías procedentes de otros terrenos intelectuales.

La propuesta de ruptura no significa que la estructura, los esquemas y los hábitos de la gestión de los medios deban ser abandonados. Las dimensiones interrelacionadas analizadas han proporcionado una importante producción especializada, así como el refinamiento de numerosas teorías previas 
que, en su conjunto, han desembocado en el relativo progreso de nuestro subcampo. Sin embargo, es oportuno, asimismo, el cuestionamiento de la estructura sobre la que se cimienta la gestión de los medios como subcampo, con el objetivo de desarrollar un nuevo catálogo de ideas y acercamientos hacia los fenómenos objeto de estudio. Una higiénica producción académica requiere un equilibrado balance "entre el desarrollo, refinamiento y explotación del conocimiento y métodos existentes y la exploración de posibles nuevas direcciones" (March, 2005). El estado de confort académico en el que se encuentra la gestión de los medios amenaza frontalmente este balance productivo en el que la trascendencia aparece en enorme desequilibrio.

En este punto es importante problematizar el proceso de conservación de la estructura tradicional y el pensamiento disruptivo, transgresor y trascendente. La ruptura trascendente no implica revolución paradigmática, algo, por otra parte, fuera de todo alcance para la mayoría de los investigadores. Mi propuesta es mucho más estrecha en contenido, esquemática en esencia y asequible en objetivos, pero que, sin embargo, proporciona ciertas claves con las que progresar en la generación de un conocimiento alternativo.

\section{Ruptura teórica}

La gestión de los medios como subcampo se configura en torno a unas estructuras ampliamente socializadas y dominadas por sus integrantes. El modelo de producción teórico se articula a través de una visión técnicopráctica, incremental y positiva. El objetivo de la ruptura teórica busca la transformación del subcampo a través de la problematización de las asunciones teóricas socializadas y la orientación hacia un ideal que hace hincapié en la trascendencia frente a lo especializado; es decir, al manejo y conocimiento de una visión teórica generalista a través del dominio de diversos terrenos intelectuales.

Frente a la adaptación acríticamente a los ideales y esquemas convencionales de la "escuela", la ruptura teórica promueve una visión externa que cuestiona la lógica dominante, así como una relación escéptica con el subcampo. La ruptura teórica, por tanto, se fundamenta en 1) el cuestionamiento y la problematización de las estructuras teóricas dominantes de 
la gestión de los medios y 2) el dominio de otros campos científicos como referencias alternativas o complementarias a la gestión de los medios. La ruptura teórica implica consecuentemente:

- El debilitamiento de los esquemas y compromisos teóricos tradicionales y el fortalecimiento de la problematización como elemento central de cuestionamiento del subcampo.

- Una relativa pérdida de identidad de sus integrantes en cuanto la especialización en gestión de medios en beneficio de una aproximación más reflexiva y amplia.

- La búsqueda de contribuciones teóricas radicales más allá del modelo incremental y el alcance de una audiencia más amplia en detrimento de la conservación tradicional del subcampo.

La ruptura teórica ambiciona una profunda apertura intelectual frente al sedentarismo académico dominante en gestión de medios. Para ello, la experimentación con otros campos teóricos que ofrezcan ideas o estilos de escritura diferentes, la ampliación de las redes sociales dominantes y el autocuestionamiento de la producción científica son elementos de fundamental importancia. La ruptura teórica, por tanto, promueve un dinamismo intelectual que "traicione" sus supuestos socializados en búsqueda de mayor trascendencia.

\section{Ruptura funcional}

La gestión de los medios toma como objeto fundamental los media en cuanto organización y heredan de la gestión pura los esquemas técnicoprácticos de evaluación de resultados. Las características definitorias y diferenciadoras de los media en cuanto organización (respecto de otro tipo de organizaciones) enmarcan la justificación del objeto como elemento configurativo del subcampo y lo dotan de relativa autonomía disciplinaria. La ruptura funcional busca la modificación del objeto "media" como organización en busca de la identificación de las estructuras y prácticas que maximicen su capacidad para sobrevivir y prosperar bajo ciertas condiciones de mercado. 
La ruptura funcional propone el objeto media como territorio de observación privilegiado para el estudio de la sociedad y sus mutaciones. El objeto media como territorio privilegiado resalta, por tanto, las diferentes dimensiones que lo modelan: gerenciales, de identidad, de poder, tecnológicos, simbólicos, ideológicos, etc. Los media no se objetivizan como organización, sino como un espacio diferenciado autónomo y válido para la discusión y reflexión sobre las mutaciones sociales y los efectos de la mediación de los propios media en la sociedad y su conjunto. La ruptura funcional busca, a partir de la especificidad de los media, la trascendencia teórica hacia otros campos con el análisis social de las características autónomas de los media, como espacio que permite el análisis de diferentes dimensiones sociales.

\section{Ruptura epistemológica}

La gestión de los medios hereda de la gestión pura su particular teoría del conocimiento en su búsqueda de la verdad. Esencialmente, la gestión de los medios parte de un acercamiento epistemológico positivista, fundamentado en la búsqueda de la verdad a través del método científico y un discurso altamente estandarizado en busca de la replicación y la razón técnico-práctica. Esta fuente que legitima el discurso científico de la gestión de los medios se fundamenta en el acatamiento de las representaciones y normas de cientificidad que debe respetar prácticamente para producir el efecto de ciencia y aspirar a la eficacia simbólica y los beneficios sociales asociados a la conformidad con las formas exteriores de la ciencia.

De esta forma, la gestión de los medios adquiere estatus de subcampo científico cuando produce un efecto de cientificidad fundado en una conformidad al menos aparente con las normas por las cuales se reconoce la ciencia. De este modo, el investigador en gestión de medios con una aproximación más interpretativa a través de un leguaje no estandarizado, incluso florido, ve amenazado su estatuto de investigador científico; por tanto, tiende a rechazar las elegancias literarias, más o menos conscientemente, y se apropia al final de los signos de cientificidad (formalismos matemáticos, ecuaciones estructurales, experimentación, etc.).Esta aproximación metodologista tiende a separar la reflexión sobre los métodos de su uso real en el trabajo científico y a cultivar el método por el método mismo. 
La ruptura epistemológica busca la emancipación de la gestión de los medios del régimen positivista dominante en las ciencias sociales como mecanismo único y absoluto de producción de "verdad”. La ruptura epistemológica se fundamenta en un politeísmo metodológico, aunque no asentado en el anarquismo epistemológico (dadaísmo) de Paul Feyerabend (1975) a través del principio del todo vale y el principio de proliferación, sino en la implementación de una batería de métodos correspondientes u orientados al problema de investigación, reflexionando constantemente sobre ellos in actu, es decir, en el mismo acto por el cual se los despliega para resolver cuestiones particulares. La ruptura epistemológica parte de un empirismo crítico, basado en el reconocimiento de la experiencia sensible como fundamento del conocimiento, pero bajo la supervisión constante de un esquema teórico superior fundamentado en el cuestionamiento, la reflexión y la crítica.

\section{Discusión y conclusiones}

Una de las ambiciones centrales del presente artículo es ofrecer una estrategia alternativa a los actuales esquemas académicos desarrollados en nuestro subcampo. Particularmente, la línea argumental de mi estudio se centra en el análisis de la estructura que configura la gestión de los medios como subcampo y que, a posteriori, lo supeditan a un problemático estado de confort académico. Como tal, entiendo una serie de posiciones, prácticas y hábitos socializados por la comunidad de gestión de medios que, a priori, profundizan aparentemente en el progreso del subcampo, pero que, sin embargo, podrían obstaculizar y oprimir el progreso académico hacia otras alternativas intelectuales más reflexivas o críticas. Diversas voces autorizadas ya han resaltado la preocupación por el futuro progreso de la gestión de los medios en cuanto a la prevalencia de determinadas epistemologías y orientaciones teóricas (Brown, 2015; Schlosberg, 2015), lo cual convierte nuestro subcampo en un espacio modélico para la implementación de nuevas ideas e interesantes contribuciones.

Como antídoto al estado de confort académico que obstaculiza el progreso alternativo de la gestión de los medios, propongo la ruptura trascendente. La ruptura trascendente ofrece un conjunto de criterios al- 
ternativos hacia el desarrollo de una investigación más imaginativa, innovadora y radical. De acuerdo con ello, he desarrollado un tipo de lenguaje que trata de capturar la esencia de la estructura de la gestión de los medios y promover un modo diferente de concebir la "buena investigación". Asimismo, he esbozado la conceptualización de ruptura teórica, ruptura funcional y ruptura epistemológica como tres de las formas fundamentales de ruptura trascendente. La ruptura teórica busca la transformación del subcampo a través del cuestionamiento y la problematización de las estructuras teóricas dominantes en la gestión de los medios, así como el dominio de otros campos científicos como referencias alternativas. La ruptura funcional busca la modificación del objeto "media" en cuanto organización y transformarlo en un espacio privilegiado para el estudio de la sociedad y sus mutaciones (gerenciales, de identidad, de poder, tecnológicos, simbólicos, ideológicos, etc.). Por último, la trascendencia epistemológica busca la emancipación de la gestión de los medios del régimen positivista dominante en las ciencias sociales como mecanismo único y absoluto de producción de "verdad" y el acercamiento a otras alternativas epistemológicas no estandarizadas.

La búsqueda de la trascendencia colisiona frontalmente con la actual estructura dominadora de la gestión de los medios. La trascendencia y una audiencia amplia deben ser inculcadas como mecanismos prácticos dentro de los estudios de doctorado de nuestro subcampo. La ampliación de los horizontes de la gestión de los medios viene de la mano de la incorporación y asimilación de una visión más amplia de lo que constituye una contribución y una revisión, posiblemente, de nuestros esquemas como escuela.

En conclusión, una de las principales implicaciones prácticas que emana de las observaciones realizadas a lo largo del presente artículo incluye: 1) la redefinición de las estructuras que configuran nuestro subcampo y que lo conducen a un aparente progreso y 2) la necesidad de profundizar en la trascendencia de nuestras contribuciones como elemento de desarrollo de nuestro subcampo. 


\section{Referencias}

Artero, J. P. (2015). Economía y empresa de comunicación: escuelas académicas y periodos de desarrollo. Austral Comunicación, 4(1), 11-40.

Alvesson, M. y Gabriel, Y. (2013). Beyond formulaic research: in prise of greater diversity in organizational research and publications. Academy of Management Learning \& Education, 12(2), 245-263.

Alvesson, M. (1987). Organization theory and technocratic consciousness. Berlín: De Gruyter.

Bourdieu, P. (1988). Homo academicus. Cambridge: Polity Press.

Bourdieu, P. (1996). Sur la Télévision. París: Liber-Raisons d’agir.

Brown, R. H. (1976). Social theory as metaphor. Theory and Society, 3(2), 169-197.

Brown, C. (2015). Media management: A critical discipline? En G. Ferrell Lowe y C. Brown, Managing media firms and industries: what's so special about media management? Londres: Springer.

Campos-Freire, F. (2010). Los nuevos modelos de gestión de las empresas mediáticas. Estudios sobre el Mensaje Periodístico, 16, 13-30.

Feyerabend, P. (1975). Against method: outline of an anarchist theory of knowledge. Londres: Humanities Press.

Goyanes, M. (2013). Estrategias y modelos de negocio: Aclaración de conceptos y terminología de la prensa en Internet. Estudios sobre el Mensaje Periodístico, 19(1), 419-431

Goyanes, M. (2015). ¿Hacia una investigación estandarizada? Observatorio $\left(\mathrm{OBS}^{*}\right)$ Journal, 9(3), 85-99. 
Kuhn, T. S. (1970). The structure of scientific revolutions. Chicago: Chicago University Press.

Küng, L. (2007). Does media management matter? Establishing the scope, rationale, and future research agenda for the discipline. Journal of Media Business Studies, 4(1), 21-39.

March, J. G. (2005). Parochialism in the evolution of a research community: the case of organization studies. Management and Organization Review, 1(1), 5-22.

Morgan, G. (1980). Paradigms, metaphors and puzzle-solving in organization theory. Administrative Science Quarterly, 25(4), 605-622.

Ortega y Gasset, J. (1929). La rebelión de las masas. Madrid: Espasa.

Schlosberg, J. (2015). Resources and perspectives from media political economy. En G. Ferrell Lowe y C. Brown, Managing media firms and industries: what's so special about media management? Londres: Springer.

Weber, M. (1946). From Max Weber: Essays in sociology. Nueva York: Oxford University Press. 